\title{
SMASIS2013-3104
}

\section{ANALYSIS-DRIVEN DESIGN OPTIMIZATION OF A SMA-BASED SLAT-COVE FILLER FOR AEROACOUSTIC NOISE REDUCTION}

\author{
William Scholten \\ Darren Hartl \\ Department of Aerospace Engineering \\ Texas A\&M University \\ College Station, Texas 77843-3409
}

\author{
Travis Turner \\ Structural Acoustics Branch \\ NASA Langley Research Center \\ Hampton, VA 23681-2199
}

\section{ABSTRACT}

Airframe noise is a significant component of environmental noise in the vicinity of airports. The noise associated with the leading-edge slat of typical transport aircraft is a prominent source of airframe noise. Previous work suggests that a slat-cove filler (SCF) may be an effective noise treatment. Hence, development and optimization of a practical slat-cove-filler structure is a priority. The objectives of this work are to optimize the design of a functioning SCF which incorporates superelastic shape memory alloy (SMA) materials as flexures that permit the deformations involved in the configuration change. The goal of the optimization is to minimize the actuation force needed to retract the slat-SCF assembly while satisfying constraints on the maximum SMA stress and on the SCF deflection under static aerodynamic pressure loads, while also satisfying the condition that the SCF self-deploy during slat extension. A finite element analysis model based on a physical bench-top model is created in Abaqus such that automated iterative analysis of the design could be performed. In order to achieve an optimized design, several design variables associated with the current SCF configuration are considered, such as the thicknesses of SMA flexures and the dimensions of various components, SMA and conventional. Designs of experiment (DOE) are performed to investigate structural response to an aerodynamic pressure load and to slat retraction and deployment. DOE results are then used to inform the optimization process, which determines a design minimizing actuator forces while satisfying the required constraints.

\section{INTRODUCTION}

In transport-class aircraft, conventional high-lift systems (e.g., leading-edge slats and trailing-edge flaps) are used to augment lift and improve stall characteristics at the low speeds required for landing. These multi-element airfoil systems nest tightly in the cruise configuration to minimize drag. The deployed multi-element airfoil system, however, presents many geometric discontinuities to the aerodynamic flow, such as edges, gaps and cavities. These geometric discontinuities are the cause for the unsteady aerodynamics that is the source for significant acoustic noise, termed airframe noise. The flow characteristics, noise production mechanisms and notional concepts for mitigation of slat noise in particular have been studied extensively. The concept of the slat-cove filler (SCF) was introduced several years ago as a possible way to fill the cavity behind the deployed slat in order to reduce the unsteadiness in the flow and, thereby, reduce the radiated acoustic noise. Various idealized versions of the SCF concept have been considered by multiple research groups and the concept has been proven, both computationally and experimentally, to be effective at reducing slat noise [1-3].

This work considers to use of shape memory alloys (SMA) materials as flexures in a leading edge SCF that can be used to reduce the aeroacoustic noise produced by the wing of a typical transport aircraft. Specifically, we perform analysis-driven design optimization of the SCF, which is currently in the developmental stage.

SMAs are attractive for use in morphing structures because 
of the large recoverable deformation that can be achieved without incurring plasticity and their high energy density. Further, the use of an SMA component can reduce the number of parts and complexity of a system [4]. With regard to aerodynamic noise reduction, the Boeing VGC [5] demonstrated how the shape memory properties of SMAs can be used to achieve noise reduction. For this work, the superelastic properties of SMAs are exploited.

Using the legacy method of smart structures design, obtaining an optimized solution required multiple iterations of designing, building, and testing physical designs. This resulted in a long and expensive cycle until an optimized solution is obtained. Due to the increasing capability of high-speed computational analysis, design problems can now be solved using optimization techniques in a more efficient way. Many of these techniques were discussed in a review of SMA design efforts [6]. Efforts have used genetic algorithms to explore the design space for applications such as SMA spring actuation components [7], SMA wire-actuated rotors [8], and SMA structural damping mechanisms [9]. Another optimization technique is the gradient-based method which has been used for applications such as an active panel structure [10]. For this work, the goal of the optimization is to minimize the activation force needed to retract the slat-SCF assembly.

This paper describes the concept of the SMA-based SCF and the process of its optimization. Specifically, Section 1 presents the physical form of the slat-SCF assembly. Section 2 addresses the computational framework and introduces the virtual model of the SCF. Section 3 discusses the goal of the optimization, the constraints, and the design parameters. Section 4 addresses the exploration of the design parameters and their influence on the model. Section 5 presents the results of the optimization. Section 6 summarizes the work and proposes concepts for future development.

\section{CONCEPT FOR SMA-BASED SLAT COVE FILLER}

As stated earlier, the goal of this work is to optimize the design of an SMA-based SCF. This proposed part fills the cavity behind the leading edge slat and guides the flow between the slat and the leading edge of the wing when the slat is deployed during low speed maneuvers [11].

Considering the amount of strain that will be needed for the SCF to be retracted (estimated to be about 2-5\% [11]), typical aerospace materials cannot be used because those materials would yield during the retraction of the SCF. In order to satisfy the deformation requirement, superelastic SMA components were considered. This type of material is assumed or chosen to be in the austenitic phase for all operating conditions and, when given a sufficient applied stress, will transform into the martensitic phase, allowing it to achieve large amounts of deformation without inducing plasticity. Once the load is removed, the SMA component will return to its original form; the deformation will be recovered as the material returns to the austenitic phase.

In a related study, a physical 2-D prototype based on a typical transport aircraft was developed [11]. The deployed and retracted configurations of the assembly can be seen in Fig. 1. The slat-SCF assembly consists of three parts; the leading edge slat, a hinge, and the SCF. The hinge is made of steel and is used to help facilitate the deployment and retraction of the SCF. The SCF can be made up of a forward SMA flexure, a steel mid-link, and an aft SMA flexure or can be formed from a monolithic strip of SMA. The slat-SCF assembly and the leading edge make up the overall leading edge assembly. When the slat-SCF assembly is in the process of retraction, the SCF will come into contact with the leading edge of the wing. This will cause an applied contact load between the SCF and the leading edge. Due to the properties of superelastic SMAs, the leading edge will cause the SCF to deform into a configuration that can fit inside the confined space between the slat and leading edge during cruise. As the slat is deployed, the SMA flexures will lose contact (thus being unloaded) with the leading edge. If the configuration is correctly designed, this will result in the SMA components returning to their original form before retraction.

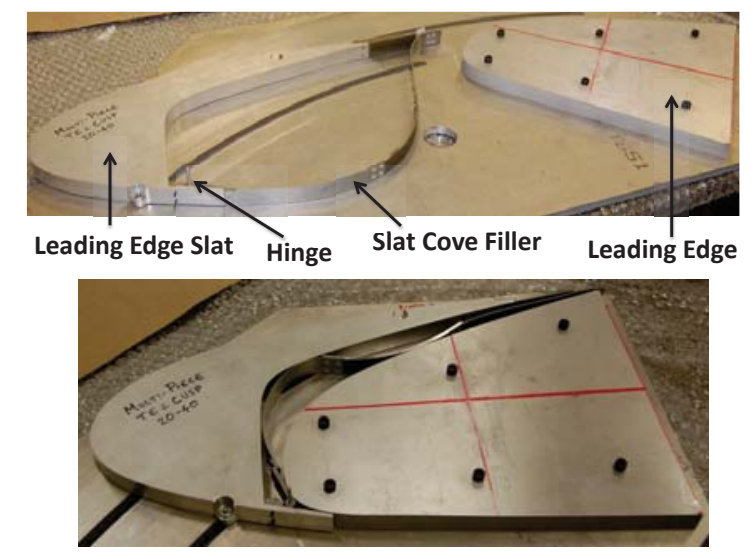

Figure 1. Picture of physical 2D model in its deployed configuration and its retracted configuration. Courtesy of [11].

\section{ANALYSIS TOOLS and FEA MODEL}

With an introduction to the proposed SMA-based SCF provided, the focus shifts to describing the computational framework that wis implemented and the analytical model of the physical assembly.

\section{Computational Framework}

To perform the analysis of different configurations of the SCF and thus obtain an optimized solution, an efficient compu- 
tational analysis framework is needed. The chosen framework uses custom constitutive model implementations in combination with a finite element suite. This combination is then automated by a simulation process management tool [6], which generates the desired finite element analysis (FEA) models and performs the analysis of those models. A post-processor is then used to obtain both local and global results of the analysis, which in turn can be used in a larger framework that assesses a design response and satisfaction of various design criteria and chooses new configurations to be analyzed.

In order to analyze the SMA flexures, a constitutive model is needed that captures the necessary mechanical and thermal responses. For this work, temperature is considered to be constant so the constitutive model must be able capture the superelastic effect of the SMA components. To do this, we have chosen the constitutive model developed by Auricchio and coworkers ( [12], [13]) . The advantage of this model is that it has been precompiled into Abaqus, allowing team members to participate in the modeling who do not have the necessary compliers to run custom-coded user material subroutines [6].

This framework is flexible, allowing various FEA tools, constitutive models, or simulation process managers to be substituted at any time. In this work, a combination of ModelCenter, Abaqus, and an Abaqus-native UMAT are used. ModelCenter is a design integration and optimization tool [14], which in this work directs the pre-processing, processing, and post-processing of Abaqus [15] through the use of custom scripts.

\section{FEA Model}

For this work, an FEA model based on the 2-D physical assembly is created and can be seen in Fig. 2. The hinge, slat, and leading edge are considered to be rigid. The SCF (a single "part" in Abaqus) is split into four sections; the SCF-hinge arm, the forward SMA flexure, the steel mid-link, and the aft SMA flexure. The partitions in the SCF are created using datum planes. This is done so that certain design parameters such as the lengths of the SMA flexures can be easily varied and the hinge arm can be extended along the curve of the SCF without modifying the hinge part. The lengths of the various SCF sections are considered as the distance that a datum plane is offset from its reference plane.

The mid-link is given material properties of steel (Young's modulus of $2000 \mathrm{ksi}$, Poisson's ratio of 0.25 , and density of $0.000732 \mathrm{slinch} / \mathrm{in}^{3}$ ) and a thickness of $0.125 \mathrm{in}$ such that it would be rigid as compared to the SMA flexures. The SCF-hinge arm is also given material properties of steel. In order to implement the Auricchio model, mechanical constants (both material properties and other parameters) must be specified. These constants are given in Table 1. The nominal values are based on typical values for superelastic SMAs. The experimental values are based on actual hysteresis loops for this SMA material provided by Johnson-Matthey Inc. The density of the SMA material is
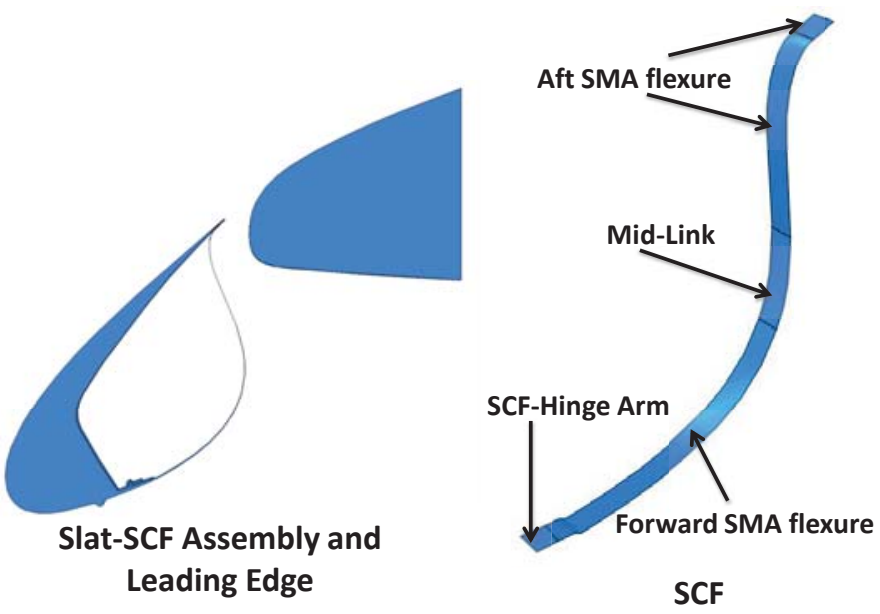

Figure 2. Analytical Model of the Leading Edge Assembly.

taken as $0.000603 \mathrm{slinch} / \mathrm{in}^{3}$. If a monolithic SMA design is desired, the length of the mid-link can be reduced to a value that would essentially neglect the mid-link.

In order to validate the experimental values, a hysteresis loop is generated in Abaqus using a two element SMA cube with the properties of Table 1. The loop is then superimposed on the experimental hysteresis loop of the SMA material which can be seen in Fig. 3. Note that the test that obtained the experimental response did not start at zero strain so the computed hysteresis loop is shifted such that both responses started at the same strain. Note also that only the first curve is of interest. As seen in this figure, the hysteresis loop from Abaqus matches the experimental response of the material quite well.

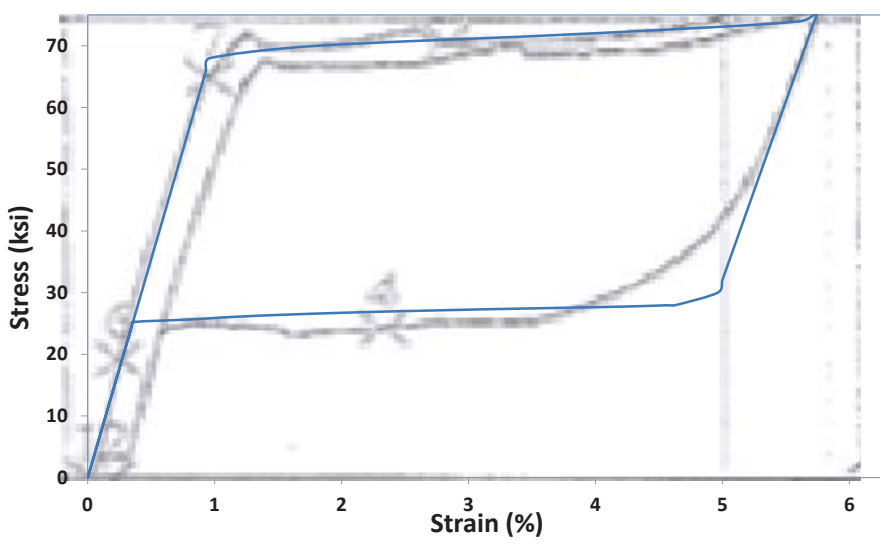

Figure 3. Abaqus generated hysteresis loop superimposed on material response from an experiment. 
Table 1. Nominal and experimental material properties used for the analysis of the SCF. Properties are associated with the Auricchio model [12], [13].

\begin{tabular}{ccc} 
Constant & Nominal & Experimental \\
\hline \multicolumn{3}{c}{ (Elastic Properties) } \\
$E_{A}$ & $7980 \mathrm{ksi}$ & $7090 \mathrm{ksi}$ \\
$E_{M}$ & $6670 \mathrm{ksi}$ & $5800 \mathrm{ksi}$ \\
$v_{A}=v_{M}{ }^{*}$ & 0.33 & 0.33 \\
\hline
\end{tabular}

(Phase Diagram Properties)

\begin{tabular}{ccc}
$\sigma^{M s}=\sigma_{c}^{M s}$ & $59 \mathrm{ksi}$ & $68.3 \mathrm{ksi}$ \\
$\sigma^{M f}$ & $74.5 \mathrm{ksi}$ & $75.1 \mathrm{ksi}$ \\
$\sigma^{A s}$ & $32.2 \mathrm{ksi}$ & $28.5 \mathrm{ksi}$ \\
$\sigma^{A f}$ & $21.5 \mathrm{ksi}$ & $24.0 \mathrm{ksi}$ \\
$C^{A}=C^{M}$ & $1070 \mathrm{psi} /{ }^{\circ} \mathrm{C}$ & $1450 \mathrm{psi} /{ }^{\circ} \mathrm{C}^{* *}$ \\
\hline
\end{tabular}

(Transformation Strain Properties)

\begin{tabular}{ccc}
$H=H_{v}$ & $5.6 \%$ & $4.4 \%$ \\
\hline \multicolumn{3}{c}{ (Other Constants) } \\
$N_{A}$ & 0 & 0 \\
$T_{0}$ & 300 & 300 \\
\hline
\end{tabular}

${ }^{*}$ Nominal values; not experimentally obtained.

**A Assumed values; not experimentally obtained.

There are two variations of the hinge and the SCF used in this study. The first variation is used in the analysis of the model response to aerodynamic loads. This variation has all three cutting planes placed horizontally with a horizontal reference plane at the forward end of the SCF. Each of the planes is offset from the plane below it in an additive scheme. For example, the "forward SMA" cutting plane is offset from the "SCF-hinge arm" cutting plane while the latter is offset from the horizontal base plane. The first variation can be seen in Fig. 4. For this variation, approximately 840 linear shell elements (type S4R) are used and are evenly distributed throughout the SCF. To analyze the model response, static analysis (a Static, General step in Abaqus/CAE) is considered and the slat is stationary.

Aerodynamic forces are considered while the SCF is fully deployed. It is assumed that these forces can be neglected when the slat-SCF assembly is in the process of being retracted or deployed. The aerodynamic forces are modeled as a distributed pressure load based on an $\mathrm{X}, \mathrm{Y}, \mathrm{Z}$ scatterplot (called a "point

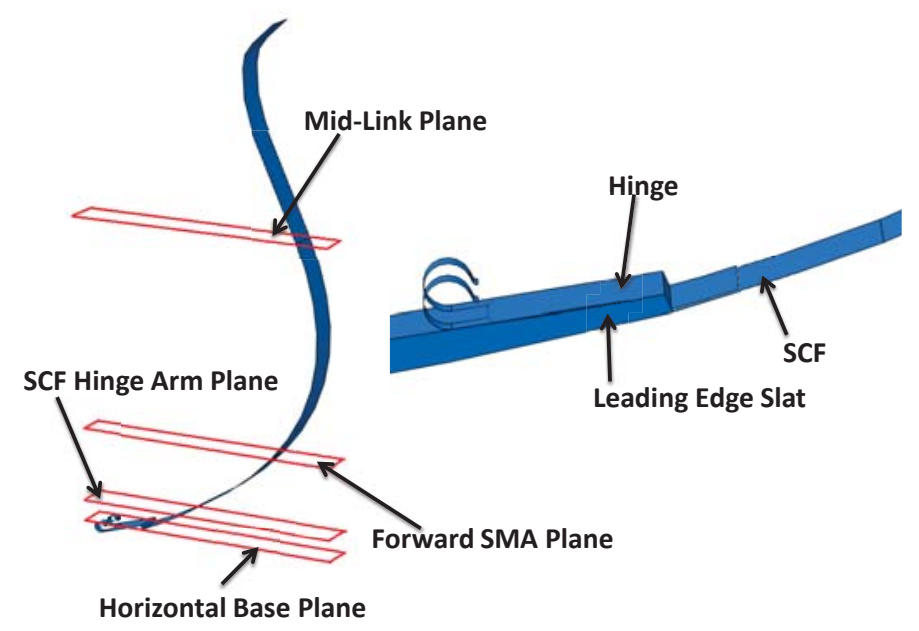

Figure 4. First Variation of the SCF and hinge.

cloud" in Abaqus) that contained a dynamic pressure value at each of a number of points in 3-D space. This point cloud is created by linearly interpolating in the U1 (chordwise, $\mathrm{X}$ ) and $\mathrm{U} 2$ (airfoil thickness, Y) direction between data points that are computationally determined by NASA aerodynamicists. The pressure is taken to be uniform in the U3 (spanwise, Z) direction. Fig. 5 shows the pressure distribution (in psi) plotted along the SCF. Also for this first variation only, the nominal properties of Table 1 are used because the experimental values had not yet been determined at the time of this analysis. Given that this is an elastic analysis with small deflections, it is assumed that the results of the analysis would not vary significantly had the experimental values been used (see $E_{A}$ and $E_{M}$ values in Table 1).

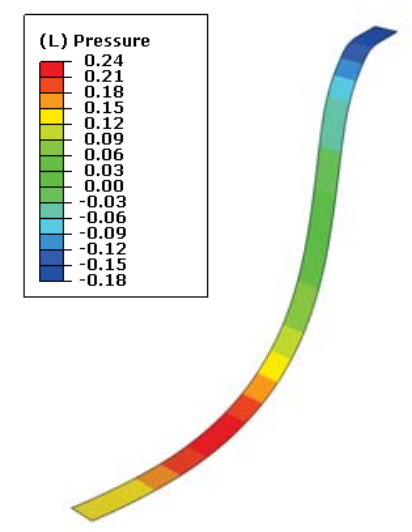

Figure 5. Distributed aerodynamic pressure load plotted along the SCF. Pressure increments are in psi. 
In order to consider configurations in which the hinge arm is smaller, the second variation of the SCF and hinge is used. For this variation, the arm of the hinge part is shortened while the forward end of the SCF is extended as seen in Figure 6. The "SCF-hinge arm" cutting plane is offset from a vertical reference plane that is positioned at forward end of the SCF. Also in this variation, the "forward SMA" cutting plane is now offset from the horizontal base plane instead of the "SCF-hinge arm" cutting plane, and is given a minimum value such that "SCF-hinge arm" cutting plane and the "forward SMA" cutting plane would not cross. The second variation of the hinge SCF is used in the retract-deploy analysis.

In order to retract and deploy the slat-SCF assembly, a hinge connector (a feature in Abaqus which connects different bodies in an assembly) is implemented between a reference point (a specified point in Abaqus) and the hinge. The reference point is then given a rotational displacement in the U3 direction such that the slat-SCF assembly would pivot about the point. In order to fully retract and deploy the assembly, a displacement of 0.57 radians is required.

While the dynamic response of the slat/SCF system during retraction and deployment is not of primary interest (i.e., only the final states are assessed), the bi-stability and associated snap-through of the SCF necessitate the use of dynamic analysis methods. For purposes of efficiency and element flexibility, the Abaqus/Standard implicit dynamic solver is chosen. A single analysis of the retract-deploy case would take approximately 3-4 hours using the previous variation. To make analysis more time efficient, the SCF is made to be one element thick with symmetry conditions in the $\mathrm{U} 3$ direction that are placed on both sides of the part, making this a "2.5-D" analysis. To reduce the required runtime even further, the contact between SCF and the other parts is converted from infinitely stiff to a linear perturbation law (still very stiff). The use of the second variation of the hinge and SCF and the one element thick constraint results in a mesh with approximately 140 linear shell elements (type S4).

\section{OPTIMIZATION PROBLEM}

Here we address the goals of the optimization, the constraints that must be satisfied, and the design parameters that will be varied in order to obtain an optimized design.

The goal of this optimization is to minimize the activation force needed to retract slat-SCF assembly. It is expected that a more compliant design will also generally correspond to a lighter design. This actuation force is measured in Abaqus as the applied moment on the reference point from which the slat-SCF assembly pivots about. This is an important criterion because the smaller the actuation force, the less power that is needed to retract the entire slat, thus making this concept more viable for eventual implementation. A secondary goal is to minimize the contact pressure between the SCF and the leading edge. If the

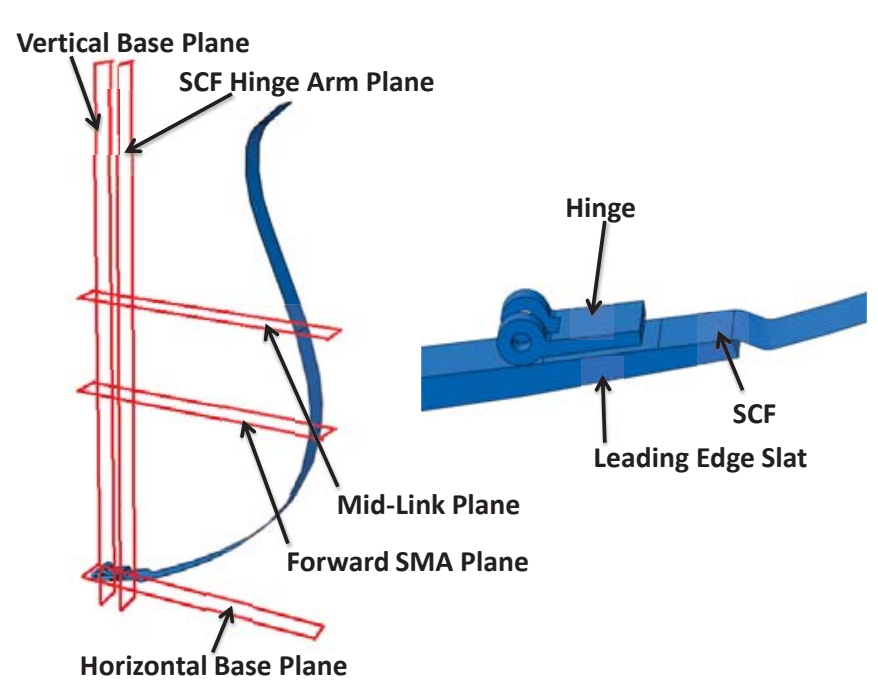

Figure 6. Second Variation of the SCF and hinge.

pressure becomes exceedingly large, the leading edge can be deformed, possibly causing losses in desired aerodynamic performance of the leading edge. Any deformation of the leading edge would make the SCF not viable for use. The parameters that are varied consist of the length of the SCF-hinge arm $\left(L_{h}\right)$, the length of the two SMA flexures $\left(L_{a f t}\right.$ and $\left.L_{\text {forward }}\right)$, and the thickness of the two SMA flexures $\left(t_{a f t}\right.$ and $\left.t_{\text {forward }}\right)$. The desired shape of the deployed SCF is taken to be fixed (i.e. determined by aerodynamicists). Therefore, one of the two SMA flexure lengths or the length of the mid-link can be directly influenced by changing the other two lengths. For this work, the aft SMA flexure is varied by varying the length of the forward SMA flexure $\left(L_{\text {forward }}\right)$ and the length of the mid-link $\left(L_{M L}\right)$.

The constraints under which this optimization are performed are that the SCF must self-deploy when the slat is rotated forward, the von Mises stress at any point in the SMA flexures does not exceed $100 \mathrm{ksi}$ during retraction, and that the SCF will be displaced no more than 0.1 in due to aerodynamic loads. If the SCF does not self-deploy, then it cannot modify the flow and is useless. Whether or not it self-deploys are determined by measuring the rotational displacement of the hinge. The stress that the SMA flexures experience must be constrained, because while the SMA components may be extremely flexible, it can still be plastically deformed due to excessive stress. If this occurs, then the desired effects of the SCF on the flow may not occur. The value of 100ksi was chosen because it is an approximate value of yield stress for this type of SMA material. The last constraint for this optimization is needed because when the slat-SCF assembly is deployed, the SCF will be subjected to aerodynamic loads. If the structure is displaced excessively, then its effect on the flow will be lessened. 


\section{INFLUENCE OF DESIGN PARAMETERS}

Before optimization could be conducted, it was necessary to perform a design of experiment (DOE) in order to understand how the design parameters effected the response of the structure to the various loads. It was decided that the aerodynamic load and the retract-deploy steps would be split into separate DOE studies so that model response to each case can be analyzed separately. Due to the amount of information that is obtained from a DOE, only the most significant results are presented here.

\section{Preliminary Aerodynamic Load DOE}

The first DOE performed assessed how the SCF was affected by the aerodynamic load while the design parameters were varied. For this DOE, the simulation process control, FEA, and UMAT components of the analysis framework were used. With the Design of Experiment feature in ModelCenter, 243 combinations of $L_{h}, t_{\text {aft }}, t_{\text {forward }}, L_{\text {forward }}$, and $L_{M L}$ were analyzed using a full factorial array. Out of the 243 runs, 60 combinations failed to converge. It was believed that these designs failed because they buckled which cannot be modeled via the approach used here. Had those runs converged, large values of displacement would have been observed. However, the remaining 183 successful runs allowed for valuable conclusions to be made about the response of the SCF to the aerodynamic load. The bounds for the parameters are shown in Table 2.

Table 2. Design variables used in the exploration of the design space for the aerodynamic load DOE. $L_{a f t}$ ranges from approximately 1.7 in to 10.1 in.

\begin{tabular}{cc}
\hline DESIGN VARIABLE & RANGE \\
\hline$L_{h}$ & $0.2-0.6 \mathrm{in}$ \\
$L_{\text {forward }}$ & $2-6 \mathrm{in}$ \\
$L_{M L}$ & $2-6 \mathrm{in}$ \\
$t_{\text {aft }}$ & $0.02-0.06 \mathrm{in}$ \\
$t_{\text {forward }}$ & $0.015-0.06 \mathrm{in}$ \\
\hline
\end{tabular}

The main outputs from this analysis were the maximum Von Mises stress in the SMA flexures and the maximum magnitude of displacement anywhere on the SCF. These two parameters define the SCF response under the aerodynamic loading.

A factor effects plot can be seen in Fig. $7^{1}$, where it was observed that the SCF thicknesses appear to have the most effect on

\footnotetext{
${ }^{1}$ Note that the failure of many runs has resulted in an unbalanced DOE, limiting the utility of this plot in the strict sense.
}

the maximum displacement while the length of the SCF-hinge arm has a negligible effect. More information about the relation between the displacement and the thicknesses can be seen in Fig. 8, which shows a ModelCenter contour plot of the displacement in terms of the two most important variables. As seen in this figure, as either thickness increased, the maximum displacement decreased. However, if one of the thicknesses was small, the other must be thick enough to compensate for the loss in stiffness. Although it was not shown, another trend that was observed was that as the mid-link length increased, the maximum displacement decreased. Clearly if more of the SCF was steel, the overall structure was stiffer, thus reducing the displacement. Of all the different combinations, no design corresponded above $10 \mathrm{ksi}$, well below the limit.

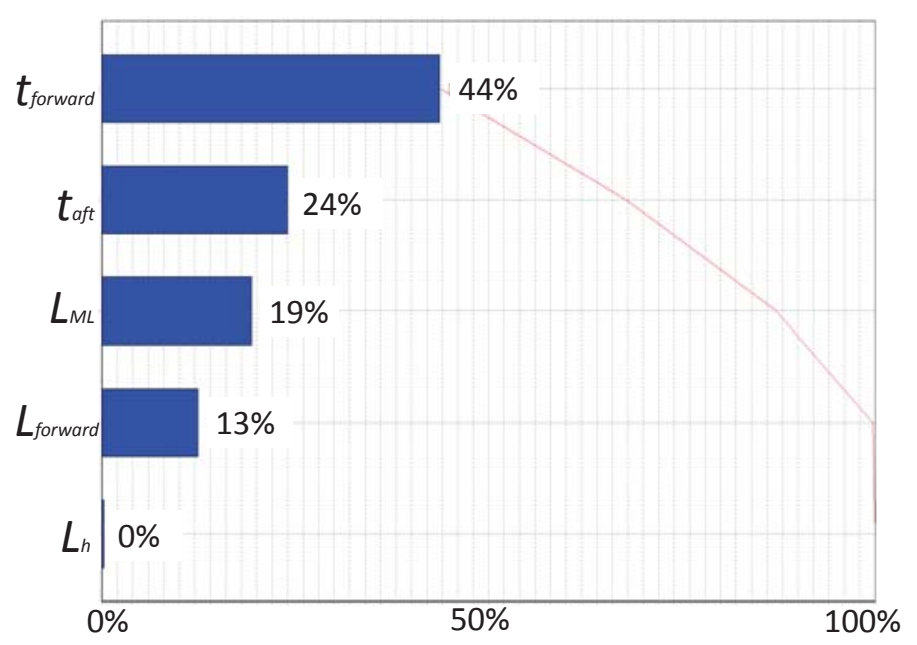

Figure 7. Influence of the design parameters on the maximum displacement of the SCF.

\section{Retract-Deploy DOE}

The second DOE considered the results of many retractdeploy analyses. Like the previous DOE, all parts of the analysis framework were used. With the Design of Experiment feature in ModelCenter, 49 combinations of $L_{h}, t_{\text {aft }}, t_{\text {forward }}, L_{\text {forward }}$, and $L_{M L}$, using a Design Explorer Orthogonal Array, were analyzed. These 49 combinations were then combined with a smaller DOE set of 25 combinations. The bounds for the parameters for both DOE studies are shown in Table 3.

The main model outputs of interest in this DOE were the rotational displacement of the hinge after deployment, the maximum applied moment needed to retract the slat-SCF assembly, the maximum von Mises stress in the SMA flexures, and the max 


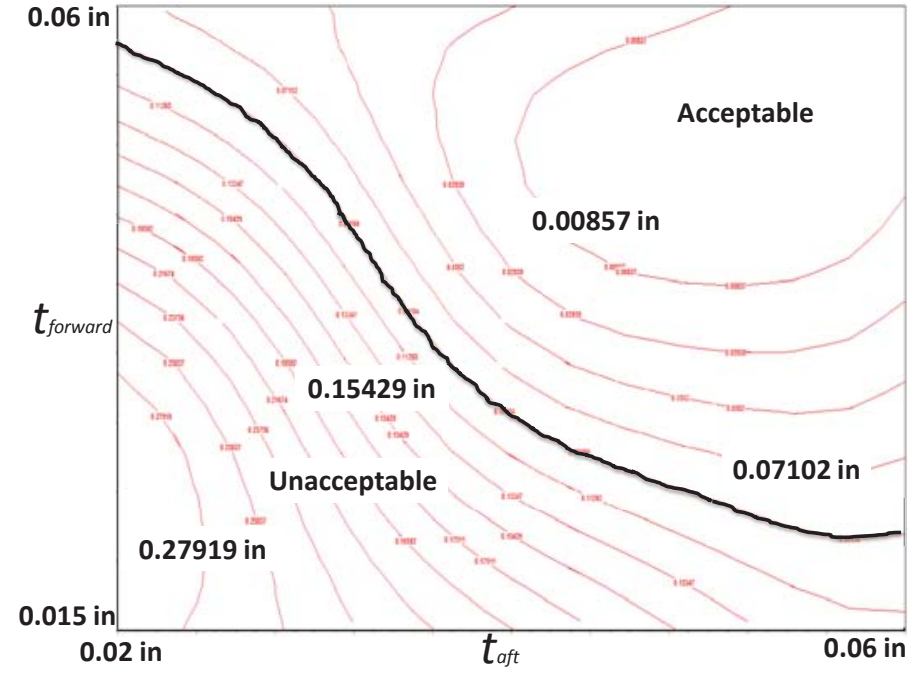

Figure 8. Contour plot showing the effect of the thicknesses on the maximum displacement $\left(L_{h}=0.4 \mathrm{in}, L_{\text {forward }}=4 \mathrm{in}, L_{M L}=4 \mathrm{in}, L_{a f t} \approx 5.9 \mathrm{in}\right)$.

Table 3. Design variables used in the exploration of physical design space for the retract-deploy analysis. $L_{a f t}$ ranges from approximately 2.3in to 12.2 in.

\begin{tabular}{cc}
\hline DESIGN VARIABLE & RANGE \\
\hline$L_{h}$ & $0.7-2$ in \\
$L_{\text {forward }}$ & $2-6 \mathrm{in}$ \\
$L_{M L}$ & $0.1-6 \mathrm{in}$ \\
$t_{\text {aft }}$ & $0.01-0.05 \mathrm{in}$ \\
$t_{\text {forward }}$ & $0.01-0.05 \mathrm{in}$ \\
\hline
\end{tabular}

contact pressure with the leading edge. Due to a computer failure, the DOE with 49 combinations was disrupted so only 31 designs were analyzed. Of these 31 combinations, 7 failed to converge. The DOE using 25 combinations also had 7 designs fail to converge. By assessing the output files associated with these runs, it was determined that analysis failure was due to a complex SCF deformation mode that is considered unacceptable. Therefore, failed runs are taken to indicate an infeasible design.

The rotational displacement of the hinge after deployment was a measure of whether or not the SCF would redeploy autonomously. It was observed that this displacement was either less than 0.1 radians (SCF redeploys) or greater than 1 radian (SCF does not redeploy). It was also observed from the DOE results that when $L_{\text {forward }}$ and $L_{M L}$ were both greater than 4in, the SCF typically did not redeploy. Based on this, one can con- clude that the aft SMA flexure drived the redeployment. If it was not large enough, the aft flexure was not able to pull the SCF out of the slat cove and back into its original form. The region of the design space corresponding to a short aft flexure should be avoided during the optimization.

Although not shown, another important result was that the length of the SCF-hinge arm had a significant effect on the maximum moment. After a particular value of the hinge-SCF arm length (estimated to be between 1.35in and 1.65in), the moment increased dramatically. This meant that large values for the length of the SCF-hinge arm should not be considered when optimizing the design. A relation between the thicknesses and the maximum moment can be observed from Fig. 9, which shows a contour plot of the maximum moment. As seen in this figure, the lower values of maximum moment typically occured at a relatively small $t_{\text {forward }}$. Lower moments at smaller thicknesses was not surprising. Smaller thicknesses meant that the SCF was less stiff so there does not have to be as much applied moment to retract the assembly. However, based on the aerodynamic load DOE, excessively small thicknesses did not satisfy the displacement constraint.

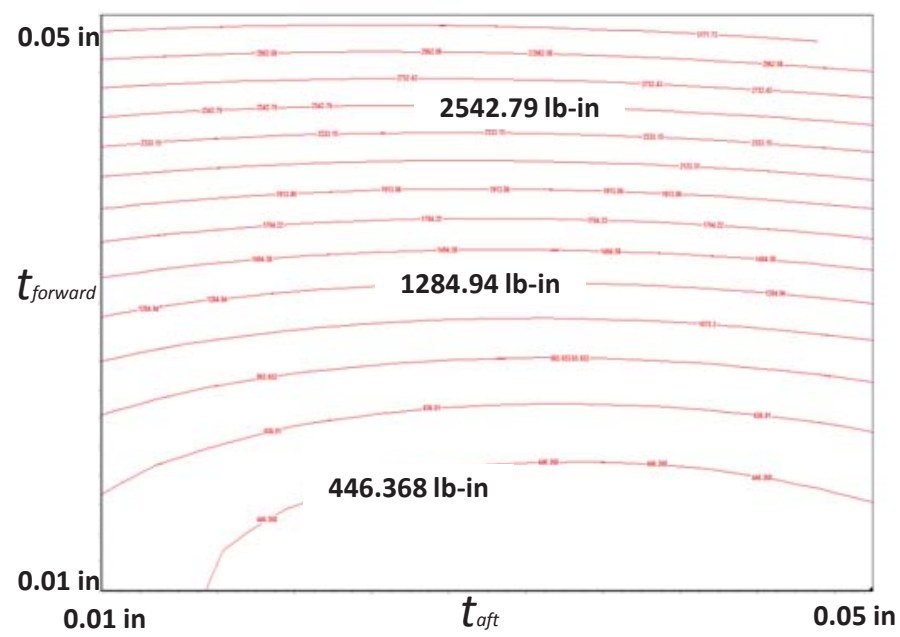

Figure 9. ModelCenter-generated contour plot showing the effect of the thicknesses on the maximum moment $\left(L_{h}=0.7 \mathrm{in}, L_{\text {forward }}=4 \mathrm{in}\right.$, $L_{M L}=0.1 \mathrm{in}, L_{a f t} \approx 10.2 \mathrm{in}$. Generated from 42 total runs).

One final relationship of interest was that of the relation between the contact pressure and maximum moment. As seen in Fig. 10 the relation between the contact pressure and the maximum moment was approximately linear. The more activation force that was applied to the slat-SCF assembly, the more load that was transferred from the SCF to the leading edge. As a re- 
sult of this relation, optimizing the SCF for the maximum applied moment will also optimize the design for the contact pressure.

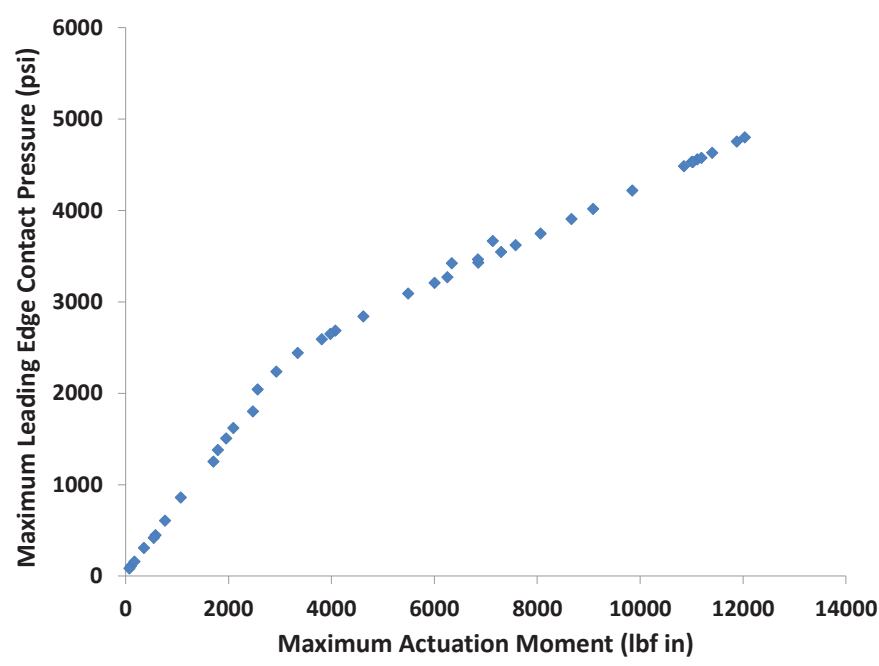

Figure 10. Relation between the maximum applied moment and the maximum contact pressure with the leading edge.

\section{OPTIMIZATION}

In order to set the bounds for the design parameters the results from both DOE studies had to be considered. The chosen bounds can be seen in Table 4. Due to the large maximum moment that results from having a large hinge arm, the upper bound of $L_{h}$ was set to $1.5 \mathrm{in}$. Changing this bound has negligible effects on model response to the aerodynamic loading as $L_{h}$ had a negligible effect on the displacement. Also, the lower bound of $L_{h}$ was decreased so that lower values of the SCF-hinge arm can be considered. The new value for the lower bound was placed at the end of the arm for the hinge part. Since the length of the aft SMA flexure was an important factor in the redeployment of the SCF, the bounds of $L_{\text {forward }}$ and $L_{M L}$ were considered at the same time. As stated earlier, when both lengths were above approximately 4in, the SCF typically failed to redeploy. To avoid this region of the design space, the upper bound of $L_{M L}$ was set to 4 in while the bounds of $L_{\text {forward }}$ were not changed. The bounds of $L_{M L}$ were chosen to be adjusted because upon further inspection of the DOE results of the retract-deploy analysis, large values of $L_{M L}$ typically corresponded to large values of stress in the midlink and a higher mass for the SCF. Also, larger values of $L_{M L}$ were more likely to result in the SCF not redeploying than larger values of $L_{\text {forward }}$. Having a smaller upper bound for $L_{M L}$ will result in designs with a larger maximum displacement. However, the constraint that the SCF redeploy is more important for this optimization, and designs can still satisfy the displacement constraint with this upper bound. The lower bound on both $t_{a f t}$ and $t_{\text {forward }}$ was increased. Based on the DOE results of the pressure analysis, a thickness of 0.01in was too thin, generally resulting in displacements greater than 0.1 in.

Table 4. Design variable ranges used for optimization. $L_{a f t}$ ranges from approximately 4 .3in to 12 .2in.

\begin{tabular}{cc}
\hline DESIGN VARIABLE & RANGE \\
\hline$L_{h}$ & $0.6-1.5 \mathrm{in}$ \\
$L_{\text {forward }}$ & $2-6 \mathrm{in}$ \\
$L_{M L}$ & $0.1-4 \mathrm{in}$ \\
$t_{\text {aft }}$ & $0.02-0.05 \mathrm{in}$ \\
$t_{\text {forward }}$ & $0.02-0.05 \mathrm{in}$ \\
\hline
\end{tabular}

Based on the results of the retract-deploy DOE, it was expected that the design that will minimize actuation moment will have the smallest thicknesses for the flexures. However, these thicknesses must not allow the SCF to fail the aerodynamic loading displacement constraint. Because of these conflicting trends, both the pressure analysis and the retract-deploy analysis were performed during the optimization. Both analyses were conducted using the same FEA model from the previous retractdeploy DOE. However the number of elements was increased to approximately 170 linear shell elements (type S4). This was done so that the number of elements along the edge of the SCF would roughly match the number of elements along the SCF edge that was used in the aerodynamic loads DOE.

To perform the optimization, the Design Explorer tool in ModelCenter was chosen( [6], [14]). The optimization process required 283 FEA runs to converge to an optimized solution. The optimized values of the design input parameters and model outputs are shown in Table 5. It is interesting to note that the optimized solution occurred at the upper boundary for $L_{M L}$. This means that a more optimized solution may exist if the steel midlink was lengthened. However, as stated earlier, that region of the design space correlates to the SCF not fully redeploying. Future work will be needed to explore this region of the design space more intensively.

\section{SUMMARY AND CONCLUSIONS}

During low speed flight, typical transport aircraft deploy high-lift systems, such as leading edge slats, in order to the improve flight characteristics of the aircraft. However when de- 
Table 5. Optimized design parameters and output

\begin{tabular}{ccc}
\hline Design Variable & Value & $\begin{array}{c}\text { Nearest } \\
\text { Bound }\end{array}$ \\
\hline SFC-hinge Length $\left(L_{h}\right)$ & 0.647 in & 0.6 in \\
Foward Flexure Length $\left(L_{\text {forward }}\right)$ & 3.28 in & 2 in \\
Mid-Link Length $\left(L_{M L}\right)$ & 4.00 in & 4 in \\
Aft Flexure Thickness $\left(t_{\text {fft }}\right)$ & 0.04625 in & 0.05 in \\
Foward Flexure Thickness $\left(t_{\text {forward }}\right)$ & 0.0238 in & 0.02 in \\
\hline
\end{tabular}

Aft Flexure Length $\left(L_{a f t}\right) \approx 7.02$ in

Maximum Displacement $=0.0991$ in

Rotational Displacement $=0$ radians

Maximum SMA von Mises Stress=70700 psi

Maximum Moment=913 lb-in

ployed, these high-lift systems present geometric discontinuities to the flow. The implementation of a SCF is a proven means by which aeroacoustic noise reduction can be achieved. The overall goal of this work was to optimize the design of a leading edge SMA-based SCF. The lengths of the hinge arm, forward SMA flexure, and mid-link and the thicknesses of the forward and aft SMA flexures were optimized in such a way that the activation force, as measured by the applied moment, would be minimized. The optimization was subject to the constraints that the von Mises stress in the SMA flexures would not exceed 100ksi, the SCF would redeploy autonomously, and that the SCF would not displace more than 0.1 in when subjected to given aerodynamic loads. A previously developed computational framework was used to perform, two DOE studies in order to investigate the model response to two different cases separately. The first DOE subjected the SCF to an aerodynamic load while it was deployed. The other DOE performed a retract-deploy analysis on the SCF. From the DOE studies, it was observed that optimizing the design for either the activation force or the contact pressure with the leading edge would optimize the design for the other, and bounds for the optimization were chosen, avoiding regions of the design space where there would not be a solution. Using ModelCenter's Design Explorer tool, both the aerodynamic load analysis and the retract-deploy analysis were performed in order to obtain an optimized solution. It was found that the optimial design occurs at the upper boundary of the mid-link length which implies that a more optimized solution may exist at larger values of $L_{M L}$.

The developments in this work have motivated future efforts such as comparing the analytical model to the physical model to in order to validate the results presented in this paper. With a validated model, further analysis such as the fluid-structure interaction and in depth CFD can be conducted. Future work may implement other constitutive models so that a more accurate representation of the SMA material can be used. In addition, other designs for the SCF shall be considered so that aerodynamic noise can be reduced in the most optimum manner.

\section{ACKNOWLEDGMENT}

The optimization and Design of Experiments were performed using a ModelCenter academic license granted by Phoenix Integration. Computational analysis was performed with an SIMULIA Abaqus research license.

\section{REFERENCES}

[1] Streett, C., Casper, J., Lockard, D., Khorrami, M., Stoker, R., Elkoby, R., Wenneman, M., and Underbrink, J., 2006. "Aerodynamic noise reduction for high-lift devices on a swept wing model". AIAA Journal(Paper 2006-212).

[2] Horne, W., James, K., Arledge, T., Soderman, P., Burnside, N., and Jaeger, S., 2005. "Measurments of 2680 foot wind tunnel". AIAA Journal(Paper 2005-2810).

[3] Imamura, T., Ura, H., Yokokawa, Y., Enomoto, S., Yamamoto, K., and Hirai, T., 2007. "Designing of slat cove filler as a noise reduction device for leading-edge slatl". AIAA Journal(Paper 2007-3473).

[4] Hartl, D. J., and Lagoudas, D., 2007. "Aerospace applications of shape memory alloys". Proceedings of the Institution of Mechanical Engineers, Part G: Journal of Aerospace Engineering, 221 (Special Issue), pp. 535-552.

[5] Mabe, J. H., Calkins, F., and Butler, G., 2006. "Boeing's variable geometry chevron, morphing aerostructure for jet noise reduction". In 47th AIAA/ ASME / ASCE / AHS / ASC Structures, Structural Dynamics and Materials Conference, pp. 1-19.

[6] Hartl, D., Lagoudas, D., and Calkins, F., 2011. “Advanced methods for the analysis, design, and optimization of SMAbased aerostructures". Smart Materials and Structures, 20(9), p. 094006.

[7] Dumont, G., and Kühl, C., 2005. "Finite element simulation for design optimisation of shape memory alloy spring actuators". Engineering Computations, 22(7), pp. $835-$ 848.

[8] Lan, C., Wang, J., and Fan, C., 2009. "Optimal design of rotary manipulators using shape memory alloy wire actuated flexures". Sensors and Actuators A: Physical, 153(2), pp. $258-266$.

[9] Ozbulut, O. E., Roschke, P. N., Lin, P. Y., and Loh, C. H., 2010. "Ga-based optimum design of a shape memory alloy 
device for seismic response mitigation". Smart Materials and Structures, 19(6), p. 065004.

[10] Widdle, Jr., R. D., Grimshaw, M. T., and Shome, M., 2009. "Optimal design of a shape memory alloy actuated composite structure with iterative finite element analysis". In Proceedings of SPIE, Smart Structures and Materials, Vol. 7288.

[11] Turner, T., Kidd, R., Hartl, D., and Scholten, W., 2013. "Development of a sma-based, slat-cove filler for reduction of aeroacoustic noise associated with transport-class aircraft wings". Proceedings of the ASME 2013 Conference on Smart Materials, Adaptive Sturctures and Intelligent Systems, Snowbird, UT.

[12] Auricchio, F., and Taylor, R. L., 1997. "Shape-memory alloys: Modelling and numerical simulation of the finitestrain superelastic behavior.". Computer Methods in Applied Mechanics and Engineering, 143(1-2), pp. 175-194.

[13] Auricchio, F., Taylor, R. L., and Lubliner, J., 1997. "Shapememory alloys: Macromodelling and numerical simulations of the superelastic behavior". Computer Methods in Applied Mechanics and Engineering, 146, pp. 281-312.

[14] ModelCenter, 2009. Model Center 9.0 User's Manual. Phoenix Integration, Philadelphia, PA.

[15] Abaqus, 2011. Analysis User's Manual. Dassault Systèmes of America Corp., Woodlands Hills, CA. 